\title{
Plant species diversity in urban areas of Northwest Siberia
}

\section{Elena Koptseva ${ }^{1}$, Olga Sumina ${ }^{1}$, Pavel Kirillov ${ }^{3}$, Alexandr Egorov2,3, and Alexandr Pechkin ${ }^{4}$}

${ }^{1}$ Department of Vegetation Science and Plant Ecology, Faculty of Biology, Saint Petersburg State University, Universitetskaya nab., 7-9,

Saint Petersburg, 199034, Russian Federation

${ }^{2}$ Department of Biogeography and Environmental Protection, Institute of Earth Sciences, Saint Petersburg State University, 10 Liniya V. O., 33-35, Saint Petersburg, 199178, Russian Federation

${ }^{3}$ S. M. Kirov Saint Petersburg State Forest Technical University, Institutskiy per., 5, Saint Petersburg, 194021, Russian Federation ${ }^{4}$ Arctic Research Center of the Yamal-Nenets Autonomous District, ul. Respubliki, 73, Salekhard, 629008, Russian Federation

Address correspondence and requests for materials to Elena Koptseva, e.koptseva@spbu.ru

\section{Abstract}

Citation: Koptseva, E., Sumina, O. Kirillov, P., Egorov, A., and Pechkin, A. 2021. Plant species diversity in urban areas of Northwest Siberia. Bio. Comm. 66(2): 129-143. https://doi.org/10.21638/ spbu03.2021.204

Authors' information: Elena Koptseva, PhD, Assistant, orcid.org/0000-0003-43879521; Olga Sumina, Dr. of Sci. in Biology, Professor, orcid.org/0000-0001-6191-228X; Pavel Kirillov, PhD student, orcid.org/00000001-7055-1922; Alexandr Egorov, PhD, Associate Professor, Head of Department, orcid.org/0000-0002-1800-0389; Alexandr Pechkin, Senior Researcher, orcid.org/00000002-6293-1252

Manuscript Editor: Pavel Skutschas, Department of Vertebrate Zoology, Faculty of Biology, Saint Petersburg State University, Saint Petersburg, Russia

Received: June 25, 2020;

Revised: October 30, 2020;

Accepted: February 18, 2021.

Copyright: (c) 2021 Koptseva et al. This is an open-access article distributed under the terms of the License Agreement with Saint Petersburg State University, which permits to the authors unrestricted distribution, and self-archiving free of charge.

Funding: The study was financially supported by the Russian Foundation for Basic Research-Yamal (Project No 19416-890002) and the State Contract of the Department of Science and Innovation of the Yamal-Nenets Autonomous District No 01-15 / 4 "Study of the processes of adaptation of deciduous and coniferous species in the Arctic and subarctic climatic zones, reclamation of disturbed lands" and the grant of Saint Petersburg State University No 28612627 "Urbanized ecosystems of the Russian Arctic: dynamics, state and sustainable development".

Ethics statement: This paper does not contain any studies involving human participants or animals performed by any of the authors.
The flora of two towns and two settlements in the northern taiga and foresttundra of Northwest Siberia (Russia) are considered. Urban species lists are limited (61-119 vascular plant species) and mainly consist of native species with a predominance of perennial herbs. Various urban functional zones (industrial, residential, recreational, vegetable patches) differ by species composition, and this difference increases in the course of city development. In the industrial zone, vegetation composition is closer to the native flora, because of the low number of adventive species. Maximal diversity is due to decorative plants, cultivars and southern weed distribution, and is typical to residential neighborhoods. Species diversity increases mainly because of woody plants introduction. Alien species are represented generally by a small number of individuals. The severe climate and poor soils limit their abilities to spread in the Far North. Urbanization forces the reduction of aboriginal biodiversity, but in northern areas where native species have the adaptive advantage, this effect is minimal. Changes in flora since 1995 were analyzed in the town of Novy Urengoy (Yamalo-Nenets Autonomous Okrug, Russia). Plant diversity increased by about $20 \%$ in all functional zones, although some alien and natural species were not recorded in 2018.

Keywords: urban environment, plant diversity, urban functional zones, urban vegetation, Arctic zone of Russian Federation

\section{Introduction}

Cities offer an unusual space to all living organisms, including plants. The various anthropogenic factors establish a large variety of habitats, where the environment selects plant species with certain ecological and biological properties. Urban conditions are favorable for short-lived plants or species well adapted to drought, but they are not conducive to the survival of insect-pollinated or wind-dispersed species, for example (Knapp, Kühn, Stolle and Klotz, 2010). Urban habitats, especially roads, often become corridors for alien and invasive species (Hao et al., 2013; Uchida et al., 2014). Control measures for invasion processes can be developed only on the basis of a comprehensive assessment of the impact of numerous environmental factors on the distribution of indigenous and alien plant species in urban ecosystems (Threlfall et al., 2016; Mavimbela, Sieben and Procheş, 2018).

In Russia, urban flora and vegetation in northern areas has been actively studied since the second half of the 20th century. The research was mainly fo- 
cused on the inventory of species diversity and process of 'synanthropization' in local flora (Trotsenko, 1990; Shushpannikova, 2001; Ishbirdin, 2004). Another aspect was the classification of urban vegetation, but it was developed only in Yakutia (Gogoleva, Cherosov and Pavlova, 1990).

Flora and plant community development in northern cities are still undergoing study and data accumulation, so the published information on these problems is fragmentary and not systematized. At the same time, the active and fast industrial development of the northern areas of Russia has caused great interest in the change of plant species diversity under the influence of urban environmental factors. The most common effect of urbanization, which is recorded by many authors, is the reduction of biodiversity of most native organisms' groups, including plants (Thompson and Jones, 1999). The flora diversity in northern cities can be increased by the introduction and acclimatization of trees and shrubs. Assortments of resistant tree and shrub species have already been recommended for the subarctic regions of Canada and Scandinavia (Smith, Poppendieck and Jensen, 2005; Riordan, Verbyla and McGuire, 2006). The use of decorative native species in northern gardening is one of the possible ways of urban floristic biodiversity support. Such work has been carried out, for example, at the ecological research station in the town of Labytnangi (Yamalo-Nenets Autonomous Okrug) since 1974 (Rozhdestvenskiy, 2000).

It is generally recognized that green spaces in residential areas make a significant contribution to the sustainability of urban ecosystems, creating a favorable environment for people (Vila-Ruiz et al., 2014). At the same time, the negative effect of planting some species which can be potentially invasive in northern areas yet seems minimal, because their acclimatization takes quite a long time. For all the above-mentioned reasons, the study of urban flora and vegetation is especially important at the present period of active industrial development in the Russian Far North.

From 1994 to 2005 the planting conditions of trees and shrubs were analyzed in numerous populated places situated in the northern taiga of Western Siberia (Khanty-Mansiysk, Surgut, Nizhnevartovsk, Beloyarsky, Tarko-Sale, Langepas, Gubkinsky) (Srodnyh, 2008). Published results not only reflect the current urban dynamics of plant species composition, but also identify a range of problems that need to be addressed as soon as possible. One of them is the inclusion of native species in urban green spaces to preserve the biodiversity of the local flora, which is very relevant.

In our opinion, the creation of effective urban greening systems in modern cities should be based on territorial zoning of the urban environment - that is, the division of urban territory into different areas (func- tional zones) performing certain functions (residential, industrial, recreational, etc.). The variety of functional zones and habitats within the town requires a different approach to their gardening, because urban vegetation must perform targeted phytomeliorative functions, and therefore must be specific to each functional zone.

Identification of the most common and active plant species - able to last for a long time in the flora of northern towns and settlements - will make it possible to effectively manage their vegetation, as well as to quickly solve any related problems via phytomelioration.

The objectives of this paper are: 1) to analyze the plant species diversity in different functional zones in several populated places of Northwest Siberia (Russia); 2) to reveal the specific features of plant species composition in different types of urban functional zones; 3 ) to study the dynamics of plant species diversity of northern urban areas in recent years.

\section{Materials and methods}

\section{Study area}

The study was carried out in 2018 on the territory of two towns - Nadym (N65'31'59.6", E72 $\left.30^{\prime} 56.0^{\prime \prime}\right)$ and Novy Urengoy (N66 $\left.05^{\prime} 19.0^{\prime \prime}, \mathrm{E} 76^{\circ} 41^{\prime} 36.6^{\prime \prime}\right)$ - and two large settlements - Pravokhettinsky (N6527'03.2", E73³1'53.6") and Pangody (N65 $\left.51^{\prime} 18.1^{\prime \prime}, \mathrm{E} 74^{\circ} 29^{\prime} 17.4^{\prime \prime}\right)$ - in the Yamalo-Nenets Autonomous Okrug, in Northwest Siberia, Russia.

The studied areas belong to different subzones (Atlas..., 2004): the northern taiga (neighborhoods of Nadym, Pravokhettinsky and Pangody) and the foresttundra (Novy Urengoy). The forest-tundra vegetation is formed mainly by open forests dominated by larch ( $\mathrm{La}$ rix sibirica Ledeb.) and spruce (Picea obovata Ledeb.), in complex with tundra and mires with a dominance of shrubs and dwarf-shrubs (i.e. Betula nana L., Empetrum nigrum subsp. hermaphroditum (Hagerup) Böcher, Ledum palustre L., Vaccinium myrtillus L., V. uliginosum L., V.vitis-idaea L.), mosses (i.e. Aulacomnium palustre (Hedw.) Schwägr., Hylocomium splendens (Hedw.) Schimp., Pleurozium schreberi (Willd. ex Brid.) Mitt., Tomentypnum nitens (Hedw.) Loeske), and lichens (i.e. Cladonia and Cetraria species). In the northern taiga, larch and spruce forests are denser, and a significant admixture of Pinus sibirica Du Tour in the tree layer is typical. The northern boundary of forests with Pinus sylvestris $\mathrm{L}$. passes through the northern taiga subzone.

The climatic conditions of the study areas are inclement. The region is influenced by continental Arctic air masses most of the year. In Nadym, despite the more southern position, the long-term mean annual temperature is about $-6.9^{\circ} \mathrm{C}$, which is lower than in Novy Urengoy $\left(-4.7^{\circ} \mathrm{C}\right)$, located to the north, in the forest-tundra. 
The average annual humidity in the northern taiga is about $75 \%$; in the forest-tundra-less than $70 \%$.

\section{Urban functional zones}

The explored towns and settlements are relatively young (Table 1). All of them received their current status in the 1970s-80s. The town of Nadym is the oldest; Pravokhettinsky settlement is the youngest.

The studied urban sites have been assigned to several socioeconomic functional zones (Table 1): industrial (A), residential neighborhoods (B), recreational (C), and vegetable patches (D). The industrial zone (A) is the territory of industrial enterprises with artificially created bulk soil. Residential neighborhoods (B) include green spaces around apartment buildings. The recreational zone $(\mathrm{C})$ is formed by parks and town gardens. In northern areas, parks are fragments of natural vegetation, located within town boundaries and adapted to the needs of residents. Artificially planted town gardens occupy small areas. Vegetable patches (D) exist only in the oldest town of Nadym, situated the furthest south of all the studied sites. This zone is a small area located in the suburbs, planted with potatoes and other crops.

The number of functional zones is connected with the age of the populated place, and only two of themthe industrial zone and residential neighborhoodswere present in all study areas (Table 1).

Table 1. Functional zones in examined towns and settlements

\begin{tabular}{l|c|c|c|c|c}
\hline \multirow{2}{*}{ Populated place } & \multirow{2}{*}{$\begin{array}{c}\text { Date of town } \\
\text { or settlement } \\
\text { status receiving }\end{array}$} & \multicolumn{4}{|c}{ Functional zone } \\
\cline { 3 - 6 } & 1972 & A & C & D \\
\hline Nadym town & 1980 & $+(9)$ & $+(4)$ & $+(4)$ & - \\
\hline Novy Urengoy town & 1979 & $+(8)$ & $+(4)$ & $+(4)$ & - \\
\hline Pangody settlement & 1984 & $+(7)$ & $+(3)$ & - & - \\
\hline $\begin{array}{l}\text { Pravokhettinsky } \\
\text { settlement }\end{array}$ & & & & & $+(15)$ \\
\hline
\end{tabular}

See text for description of functional zones. The number of study plots is shown in parentheses.

\section{Field data collection}

In all populated places the vascular plant species were listed separately for each functional zone on the base of vegetation descriptions on $25 \mathrm{~m}^{2}$ plots, 73 in total. Moreover, species lists were supplemented by rare and low-abundant species that were not present in the plots. For each sample plot, the following parameters were registered: community type (woody, shrubs, herbaceous), total vegetation cover (\%), cover of trees, shrubs, dwarfshrubs, herbs, graminoids, mosses, lichens, as well as cover for each species.
Latin plant names were standardized according to the International Plant Name Index (IPNI) (https:// www.ipni.org) (date of request -27.11 .2020 ).

\section{Data processing}

Lists of vascular plant species (138 in total) as well as the lists of species in each of the four types of functional zones in towns and settlements were compiled. The Jaccard index was calculated to estimate the similarity of plant species composition in different urban functional zones.

Indirect ordination of sample plots was carried out by nonmetric multidimensional scaling (NMDS) using Bray-Curtis Analysis in $\mathrm{R}$ statistical software, version 3.5.2, to identify the differences in vegetation of urban functional zones.

\section{Results}

\section{Plant species diversity in urban functional zones}

The flora of the four observed towns and settlements includes 138 species of vascular plants from 29 families (Table 2). The greatest number of species was found in Novy Urengoy (119 species); 104 species in Nadym; 85 in Pangody; and 61 in Pravokhettinsky. Non-woody plants predominate in all populated places. Perennial herbs form a significant part (70\%) of the total list. Among them, the Poaceae family, with a total of 27 species, leads in the number of species in all types of urban functional zones. A wide variety of graminoids is associated with the presence of cultivars in grass mixtures used for lawns or artificial restoration of disturbed areas in northern populated places. Graminoids also play the main role in natural recovery of various urban waste lands. There are also many species in the Asteraceae and Salicaceae families. Other families are represented by fewer species.

Woody plants (trees and shrubs) are represented by 30 taxa attributed to 7 families. It is about only $20 \%$ of the total species list. The largest number of woody species is in Salicaceae (13 species) (Fig. 1).

Planted alien woody species dominate in residential neighborhoods. Industrial zones in all populated places are occupied only by native species of woody plants such as Duschekia fruticosa (Rupr.) Raus, Salix bebbiana Sarg., S. gmelinii Pall., S.lapponum L., S. phylicifolia L., S. viminalis L., Sorbus sibirica (Hedl.) Krylov. Currently, the proportion of alien woody plants used in the greenery of northern places is less than $20 \%$ (only 6 taxa) of the total number of tree and shrub species.

Of urban flora, $26 \%$ of species are shrubs and dwarf-shrubs. More than half of them (63\%) are native species. Indigenous willows (i.e. Salix bebbiana, S. gmelinii, S. lapponum, S. phylicifolia, S. viminalis) are the most 
Table 2. The list of vascular plant species recorded in various urban functional zones

\begin{tabular}{|c|c|c|c|c|c|c|c|c|c|c|c|c|c|c|}
\hline \multirow{2}{*}{ Family and species names } & \multirow{2}{*}{ CG } & \multirow{2}{*}{ Range } & \multicolumn{4}{|c|}{ Nadym } & \multicolumn{3}{|c|}{ N. Urengoy } & \multicolumn{3}{|c|}{ Pangody } & \multicolumn{2}{|c|}{ Pravok } \\
\hline & & & A & B & $\mathrm{C}$ & $\mathrm{D}$ & $A$ & B & $\mathrm{C}$ & $A$ & B & C & A & B \\
\hline Equisetaceae & & & 1 & 2 & 2 & 1 & 1 & 2 & 2 & 1 & 2 & 2 & 2 & 1 \\
\hline Equisetum arvense & W-S & $\mathrm{b}$ & + & + & + & + & + & + & + & + & + & + & + & + \\
\hline *E. pratense & $\mathrm{F}$ & $a b$ & & + & + & & & + & + & & + & + & + & \\
\hline Pinaceae & & & 1 & 2 & 3 & 0 & 1 & 3 & 3 & 1 & 3 & 2 & 2 & 3 \\
\hline Larix sibirica & $\mathrm{F}$ & $b$ & + & + & + & & + & + & + & + & + & + & + & + \\
\hline Picea obovata & $\mathrm{F}$ & $\mathrm{b}$ & & & + & & & + & + & & + & + & & + \\
\hline Pinus sibirica & $\mathrm{F}$ & $b$ & & + & + & & & + & + & & + & & + & + \\
\hline Poaceae & & & 6 & 9 & 7 & 4 & 13 & 20 & 9 & 8 & 11 & 8 & 6 & 9 \\
\hline${ }^{*}$ Agrostis tenuis & M & b & & + & + & & & + & & & & + & & + \\
\hline *Alopecurus aequalis & W-S & $b$ & & & & & + & + & & + & + & & & \\
\hline *A. pratensis & M & $b$ & & & & & & + & & & & & & \\
\hline Avena sativa & CUL & $\mathrm{pl}$ & & & & & & + & & & & & & \\
\hline *Beckmannia syzigachne & W-S & $\mathrm{b}$ & & & & & + & & & + & + & & & \\
\hline Bromopsis inermis & M & $b$ & & + & + & & & & & & + & + & & \\
\hline *Calamagrostis epigeios & $\mathrm{F}$ & b & & + & & & + & + & & + & + & + & + & \\
\hline${ }^{*}$ C. langsdorffii & W-S & $a b$ & & + & + & & + & & & & & & & \\
\hline *C. lapponica & $\mathrm{T}$ & ha-m & + & & & & + & & + & & & & + & \\
\hline${ }^{*}$ C. purpurea & W-S & $a b$ & + & + & + & & + & + & & + & + & & + & + \\
\hline *Dactylis glomerata & M & $\mathrm{b}$ & & + & & & & + & + & & & & & + \\
\hline *Deschampsia borealis & $\mathrm{T}$ & a & + & & & & & + & & + & + & + & + & + \\
\hline D. obensis & $\mathrm{T}$ & ha & + & & & & + & + & + & & & & & \\
\hline *Elytrigia repens & M & $\mathrm{b}$ & & + & & + & & + & & & & + & & + \\
\hline *Festuca ovina & $\mathrm{F}$ & $a b$ & + & + & + & + & + & + & + & + & + & + & + & + \\
\hline *F. pratensis & M & $\mathrm{b}$ & & & & & & + & & & & & & \\
\hline${ }^{*}$ F. rubra & M & $a b$ & & & + & & + & + & + & & + & + & & + \\
\hline *Hierochloë alpina & $\mathrm{T}$ & aa & & & & & & & + & & & & & \\
\hline *Hordeum jubatum & $\mathrm{R}$ & $\mathrm{b}$ & & & & & + & & & & & & & \\
\hline Lolium perenne & CUL & CUL & & & & & & + & & & & & & \\
\hline *Phleum pratense & M & $\mathrm{b}$ & & + & & + & & + & & & + & & & \\
\hline *Poa alpigena & $T$ & a & + & & & & + & + & + & + & & & + & \\
\hline$* P$. arctica & $\mathrm{T}$ & a & & & & & + & & + & & & & & \\
\hline *P. pratensis & M & $a b$ & & & + & & & + & & & + & + & & + \\
\hline *Puccinellia distans & $\mathrm{R}$ & $b$ & & & & + & & + & & + & + & & & + \\
\hline *P. hauptiana & $\mathrm{R}$ & b & & & & & + & + & + & & & & & \\
\hline Triticum aestivum & CUL & $\mathrm{pl}$ & & & & & & + & & & & & & \\
\hline Cyperaceae & & & 6 & 0 & 1 & 0 & 5 & 2 & 4 & 6 & 1 & 0 & 4 & 1 \\
\hline *Eriophorum angustifolium & W-S & $a b$ & + & & & & + & & & + & & & & + \\
\hline E. scheuchzeri & W-S & aa & + & & & & + & & & & & & & \\
\hline Carex aquatilis & W-S & $\mathrm{b}$ & + & & & & + & & & + & & & + & \\
\hline C. arctisibirica & $T$ & a & & & & & + & + & + & + & & & & \\
\hline C. bigelowii & T & a & + & & + & & & & + & & & & + & \\
\hline C. canescens & W-S & b & + & & & & + & + & & + & & & + & \\
\hline C. globularis & $\mathrm{F}$ & $b$ & & & & & & & + & + & & & + & \\
\hline C. nigra & W-S & $\mathrm{b}$ & + & & & & & & + & + & + & & & \\
\hline
\end{tabular}




\begin{tabular}{|c|c|c|c|c|c|c|c|c|c|c|c|c|c|c|}
\hline \multirow{2}{*}{ Family and species names } & \multirow{2}{*}{ CG } & \multirow{2}{*}{ Range } & \multicolumn{4}{|c|}{ Nadym } & \multicolumn{3}{|c|}{ N. Urengoy } & \multicolumn{3}{|c|}{ Pangody } & \multicolumn{2}{|c|}{ Pravok } \\
\hline & & & A & B & C & D & A & B & C & A & $\mathrm{B}$ & $\mathrm{C}$ & A & B \\
\hline Juncaceae & & & 3 & 1 & 0 & 0 & 5 & 0 & 3 & 1 & 0 & 1 & 4 & 2 \\
\hline *Juncus arcticus & W-S & ha-m & & & & & + & & & & & & & \\
\hline J. filiformis & W-S & $b$ & + & & & & + & & & & & + & + & + \\
\hline *J. trifidus & $\mathrm{T}$ & aa & + & & & & + & & + & + & & & + & + \\
\hline *Luzula confusa & $\mathrm{T}$ & aa & & & & & + & & + & & & & + & \\
\hline *L. multiflora & M & $\mathrm{b}$ & + & + & & & + & & + & & & & + & \\
\hline Salicaceae & & & 8 & 6 & 5 & 0 & 4 & 8 & 8 & 5 & 3 & 5 & 6 & 6 \\
\hline Populus tremula & $\mathrm{F}$ & $b$ & & + & + & & & + & + & & + & + & & \\
\hline Salix bebbiana & $\mathrm{F}$ & $b$ & + & + & + & & & + & + & & & + & & \\
\hline *S. caprea & $\mathrm{F}$ & $b$ & + & + & + & & & + & + & & + & & & \\
\hline S. glauca & $\mathrm{T}$ & ha-m & + & & & & & & + & & & & + & + \\
\hline S. gmelinii & W-S & b & + & + & & & + & + & + & + & & + & + & + \\
\hline S. jenisseensis & CUL & CUL & & + & + & & & & & & & & & \\
\hline S. lanata & $\mathrm{T}$ & ha-m & + & & & & & & + & + & & & + & + \\
\hline S. lapponum & $\mathrm{T}$ & $a b$ & + & & & & + & + & + & + & & & + & \\
\hline *S. myrsinifolia & $\mathrm{F}$ & $b$ & & & + & & & & & & & + & & + \\
\hline S. myrtilloides & $\mathrm{T}$ & $b$ & + & & & & & + & & & & & & \\
\hline S. phylicifolia & W-S & $a b$ & + & & & & + & + & & + & & + & + & + \\
\hline S. saposhnikovii & CUL & CUL & & + & & & & & & & & & & \\
\hline *S. viminalis & W-S & $b$ & & & & & + & + & + & + & + & & + & + \\
\hline Betulaceae & & & 4 & 3 & 4 & 0 & 2 & 6 & 5 & 3 & 2 & 1 & 1 & 3 \\
\hline Betula $\mathrm{x}$ aurata & CUL & CUL & + & + & + & & & + & + & & & & & \\
\hline *Betula nana & $\mathrm{T}$ & ha & & & + & & + & + & + & + & & & + & \\
\hline B. pendula & $\mathrm{F}$ & $b$ & + & + & + & & & + & + & & & & & + \\
\hline B. pubescens & $\mathrm{F}$ & $b$ & + & + & + & & & + & & + & + & + & & + \\
\hline B. tortuosa & $\mathrm{F}$ & ab-m & & & & & + & + & + & & & & & \\
\hline Duschekia fruticosa & $\mathrm{F}$ & ab-m & + & & & & & + & + & + & + & & & + \\
\hline Polygonaceae & & & 2 & 3 & 1 & 2 & 3 & 3 & 1 & 2 & 1 & 1 & 2 & 2 \\
\hline Aconogonon ocreatum var. laxmannii & T & ha & & & & & + & & & & & & & \\
\hline *Rumex acetosella & M & $\mathrm{pl}$ & + & + & & + & & + & & + & & & + & + \\
\hline${ }^{*}$. graminifolius & M & ha & & & & & + & + & & & & & & \\
\hline *Polygonum aviculare & $\mathrm{R}$ & $\mathrm{pl}$ & & + & & & & & & & & & & \\
\hline *P. humifusum & $\mathrm{R}$ & ha & + & + & + & + & + & + & + & + & + & + & + & + \\
\hline Chenopodiaceae & & & 1 & 0 & 0 & 1 & 1 & 1 & 0 & 0 & 0 & 0 & 0 & 0 \\
\hline *Chenopodium album & $\mathrm{R}$ & $\mathrm{pl}$ & + & & & + & + & + & & & & & & \\
\hline Caryophyllaceae & & & 1 & 3 & 1 & 1 & 3 & 5 & 2 & 1 & 0 & 2 & 1 & 1 \\
\hline *Sagina intermedia & M & a & & & & & + & + & & & & & & \\
\hline Silene paucifolia & M & a & + & + & + & & + & + & + & & & + & + & + \\
\hline *Stellaria ciliatosepala & W-S & ha & & & & & + & + & + & + & & & & \\
\hline *S. graminea & M & $b$ & & + & & & & + & & & & + & & \\
\hline *S. media & $\mathrm{R}$ & $\mathrm{pl}$ & & + & & + & & + & & & & & & \\
\hline Ranunculaceae & & & 0 & 1 & 0 & 1 & 0 & 1 & 0 & 0 & 1 & 0 & 1 & 1 \\
\hline *Ranunculus repens & $\mathrm{F}$ & $b$ & & + & & + & & + & & & + & & + & + \\
\hline
\end{tabular}




\begin{tabular}{|c|c|c|c|c|c|c|c|c|c|c|c|c|c|c|}
\hline \multirow{2}{*}{ Family and species names } & \multirow{2}{*}{ CG } & \multirow{2}{*}{ Range } & \multicolumn{4}{|c|}{ Nadym } & \multicolumn{3}{|c|}{ N. Urengoy } & \multicolumn{3}{|c|}{ Pangody } & \multicolumn{2}{|c|}{ Pravok } \\
\hline & & & A & B & C & $\mathrm{D}$ & A & B & $\mathrm{C}$ & A & B & C & A & B \\
\hline Brassicaceae & & & 4 & 4 & 0 & 2 & 3 & 6 & 0 & 3 & 2 & 2 & 4 & 3 \\
\hline *Barbarea vulgaris & M & $\mathrm{b}$ & + & & & & & + & & & & & + & \\
\hline *Berteroa incana & M & $\mathrm{pl}$ & & + & & & & & & & & & & \\
\hline *Capsella bursa-pastoris & M & $\mathrm{pl}$ & & + & & + & & + & & & & + & & \\
\hline *Descurainia sophioides & M & ha & + & & & & + & + & & + & + & + & + & + \\
\hline *Erysimum cheiranthoides & M & $b$ & + & & & & + & + & & + & + & & + & + \\
\hline *Raphanus raphanistrum & M & $\mathrm{pl}$ & & + & & + & & + & & & & & & \\
\hline *Rorippa palustris & W-S & $\mathrm{pl}$ & + & + & & & + & + & & + & & & + & + \\
\hline Parnassiaceae & & & 1 & 0 & 0 & 0 & 1 & 1 & 0 & 1 & 0 & 0 & 0 & 0 \\
\hline Parnassia palustris & W-S & $\mathrm{b}$ & + & & & & + & + & & + & & & & \\
\hline Grossulariaceae & & & 0 & 0 & 0 & 0 & 0 & 1 & 0 & 0 & 0 & 0 & 0 & 0 \\
\hline Ribes spicatum & CUL & CUL & & & & & & + & & & & & & \\
\hline Rosaceae & & & 1 & 5 & 2 & 0 & 0 & 4 & 1 & 1 & 2 & 3 & 1 & 2 \\
\hline Padus avium ssp. borealis & $\mathrm{F}$ & ha-m & + & + & + & & & + & + & & & & & \\
\hline *Potentilla norvegica & M & $\mathrm{pl}$ & & + & & & & & & & & + & & \\
\hline Rosa acicularis & $\mathrm{F}$ & $a b$ & & + & & & & + & & + & + & + & & + \\
\hline Rubus idaeus & $\mathrm{F}$ & $b$ & & + & & & & + & & & & & & \\
\hline Sorbus sibirica & $\mathrm{F}$ & b-m & & + & + & & & + & & & + & + & + & + \\
\hline Fabaceae & & & 2 & 3 & 3 & 1 & 0 & 3 & 0 & 0 & 3 & 5 & 1 & 4 \\
\hline *Medicago sativa & M & $b$ & & + & & & & + & & & + & + & + & + \\
\hline *Melilotus albus & M & $\mathrm{pl}$ & & + & + & & & & & & & + & & + \\
\hline *Trifolium pratense & M & b & + & & + & & & + & & & + & + & & \\
\hline${ }^{*} T$. repens & M & $b$ & + & & + & + & & + & & & + & + & & + \\
\hline *Vicia cracca & M & $\mathrm{b}$ & & + & & & & & & & & + & & + \\
\hline Oleaceae & & & 0 & 1 & 1 & 0 & 0 & 2 & 1 & 0 & 0 & 0 & 0 & 0 \\
\hline Syringa $\mathrm{x}$ henryi & CUL & CUL (nm) & & + & + & & & + & + & & & & & \\
\hline Syringa josikaea & CUL & CUL (nm) & & & & & & + & & & & & & \\
\hline Empetraceae & & & 0 & 0 & 0 & 0 & 0 & 0 & 1 & 1 & 0 & 0 & 1 & 0 \\
\hline Empetrum hermaphroditum & $\mathrm{T}$ & ha-m & & & & & & & + & + & & & + & \\
\hline Onagraceae & & & 2 & 1 & 1 & 1 & 2 & 2 & 1 & 2 & 1 & 1 & 1 & 1 \\
\hline *Chamaenerion angustifolium & $\mathrm{F}$ & ab-m & + & + & + & + & + & + & + & + & + & + & + & + \\
\hline *Epilobium palustre & W-S & $a b$ & + & & & & + & + & & + & & & & \\
\hline Pyrolaceae & & & 0 & 0 & 1 & 0 & 0 & 0 & 0 & 0 & 0 & 0 & 0 & 0 \\
\hline Pyrola minor & $\mathrm{F}$ & $a b$ & & & + & & & & & & & & & \\
\hline Ericaceae & & & 0 & 0 & 3 & 0 & 2 & 0 & 3 & 4 & 0 & 0 & 0 & 0 \\
\hline Arctostaphylos uva-ursi & $\mathrm{F}$ & $b$ & & & + & & & & & & & & & \\
\hline Arctous alpina & $\mathrm{T}$ & ha-m & & & + & & & & + & + & & & & \\
\hline Ledum palustre & W-S & $a b$ & & & & & & & & + & & & & \\
\hline Vaccinium vitis-idaea & $\mathrm{F}$ & $b$ & & & + & & + & & + & + & & & & \\
\hline V. uliginosum & W-S & $\mathrm{b}$ & & & & & + & & + & + & & & & \\
\hline Primulaceae & & & 0 & 0 & 1 & 0 & 0 & 0 & 0 & 0 & 0 & 1 & 0 & 0 \\
\hline Trientalis europaea & $\mathrm{F}$ & $a b$ & & & + & & & & & & & + & & \\
\hline Lamiaceae & & & 0 & 0 & 0 & 1 & 0 & 0 & 0 & 0 & 0 & 0 & 0 & 0 \\
\hline Origanum vulgare & CUL & CUL & & & & + & & & & & & & & \\
\hline
\end{tabular}


End of Table 2

\begin{tabular}{|c|c|c|c|c|c|c|c|c|c|c|c|c|c|c|}
\hline \multirow{2}{*}{ Family and species names } & \multirow{2}{*}{ CG } & \multirow{2}{*}{ Range } & \multicolumn{4}{|c|}{ Nadym } & \multicolumn{3}{|c|}{ N. Urengoy } & \multicolumn{3}{|c|}{ Pangody } & \multicolumn{2}{|c|}{ Pravok } \\
\hline & & & A & B & $\mathrm{C}$ & $\mathrm{D}$ & A & B & $\mathrm{C}$ & A & B & $\mathrm{C}$ & $A$ & B \\
\hline Solanaceae & & & 0 & 0 & 0 & 0 & 0 & 1 & 0 & 0 & 0 & 0 & 0 & 0 \\
\hline Solanum tuberosum & CUL & $\mathrm{pl}$ & & & & & & + & & & & & & \\
\hline Scrophulariacea & & & 2 & 3 & 0 & 1 & 2 & 3 & 1 & 1 & 1 & 1 & 1 & 2 \\
\hline *Euphrasia frigida & W-S & a & + & + & & & + & + & & + & & & + & + \\
\hline *Linaria vulgaris & M & $b$ & & + & & + & & & & & & & & + \\
\hline Pedicularis labradorica & W-S & ha-m & & & & & & & + & & & & & \\
\hline${ }^{*}$ Rhinanthus vernalis & M & $\mathrm{b}$ & + & & & & + & + & & & & + & & \\
\hline Veronica longifolia & M & $a b$ & & + & & & & + & & & + & & & \\
\hline Plantaginaceae & & & 0 & 1 & 0 & 0 & 0 & 0 & 0 & 0 & 0 & 0 & 0 & 1 \\
\hline *Plantago major & M & $\mathrm{b}$ & & + & & & & & & & & & & + \\
\hline Caprifoliaceae & & & 0 & 1 & 0 & 0 & 0 & 1 & 1 & 0 & 0 & 0 & 0 & 0 \\
\hline Lonicera caerulea ssp. pallasii & $\mathrm{F}$ & $\mathrm{bm}$ & & + & & & & + & + & & & & & \\
\hline Campanulaceae & & & 0 & 1 & 1 & 0 & 1 & 1 & 0 & 0 & 0 & 1 & 0 & 0 \\
\hline Campanula rotundifolia & M & $a b$ & & + & + & & + & + & & & & + & & \\
\hline Asteraceae & & & 9 & 9 & 6 & 4 & 8 & 14 & 4 & 10 & 8 & 7 & 5 & 3 \\
\hline *Achillea millefolium & M & $\mathrm{b}$ & & + & & & & + & & & + & + & & \\
\hline Antennaria dioica & $\mathrm{F}$ & $a b$ & & & + & & & & + & + & & & & \\
\hline${ }^{*}$ Artemisia tilesii & $\mathrm{T}$ & a & & & & & + & + & & + & & & & \\
\hline A. vulgaris & $\mathrm{R}$ & $\mathrm{b}$ & + & & & & & + & & + & + & + & & \\
\hline${ }^{*}$ Crepis tectorum & M & $\mathrm{b}$ & + & & + & + & + & + & & + & + & & & \\
\hline Erigeron acris & M & a & + & & & & & + & & & + & & + & \\
\hline *Helianthus annuus & CUL & $\mathrm{pl}$ & & + & & & & & & & & & & \\
\hline *Hieracium umbellatum & $\mathrm{F}$ & $b$ & + & + & + & + & + & + & + & + & + & + & + & + \\
\hline *Lactuca sibirica & $\mathrm{F}$ & $\mathrm{b}$ & + & + & & & & + & & & + & + & & \\
\hline Matricaria discoidea & M & $b$ & & & & & & + & & & & & & \\
\hline M. recutita & M & $\mathrm{pl}$ & & + & & & & + & & & & & & \\
\hline *Petasites frigidus & W-S & $a b$ & & & & & + & & & & & & & \\
\hline Solidago lapponica & $\mathrm{F}$ & ha & & & & + & & & + & + & & & & \\
\hline *Tanacetum bipinnatum & M & ha & & + & + & & + & + & & + & & + & & \\
\hline T. vulgare & $\mathrm{R}$ & b & & & & & & + & & & + & & & \\
\hline *Taraxacum ceratophorum & M & ha-m & + & + & + & & & + & & & & + & + & + \\
\hline Tephroseris palustris & W-S & $a b$ & & & & & + & + & & + & & & & \\
\hline *Tripleurospermum hookeri & $\mathrm{R}$ & a & + & + & + & + & + & + & + & + & & + & + & + \\
\hline T. inodorum & $\mathrm{R}$ & $b$ & + & + & & & + & & & & & & & \\
\hline *Tussilago farfara & W-S & $a b$ & + & & & & & & & + & + & & + & \\
\hline $\begin{array}{l}\text { Total number of species: } \\
\text { in functional zone } \\
\text { among them apophyte species }\end{array}$ & & & $\begin{array}{l}54 \\
28 \\
\end{array}$ & $\begin{array}{l}59 \\
35\end{array}$ & $\begin{array}{l}43 \\
20\end{array}$ & $\begin{array}{l}20 \\
17\end{array}$ & $\begin{array}{l}59 \\
38\end{array}$ & $\begin{array}{l}90 \\
51\end{array}$ & $\begin{array}{l}50 \\
20\end{array}$ & $\begin{array}{l}51 \\
30 \\
\end{array}$ & $\begin{array}{l}41 \\
26\end{array}$ & $\begin{array}{l}43 \\
27\end{array}$ & $\begin{array}{l}43 \\
26\end{array}$ & $\begin{array}{l}45 \\
30\end{array}$ \\
\hline in town or settlement & & & & & & & & 119 & & & 85 & & & \\
\hline
\end{tabular}

Functional zones: A — industrial, B — residential neighborhoods, C - recreational, D - vegetable patches. Range: $n$ m - Nemoral; b - Boreal, ab - Arctoboreal, ab-m - Arctoboreal-Montane, bm -Boreal-Montane; ha - Hypoarctic, ha-m - Hypoarctic-Montane, a - Arctic, aa - ArctoAlpine; pl - Plurizonal. Coenotic group (CG): W-S - water-swamp, F - forest, M - meadow, T - tundra, R - ruderal habitats. Other abbreviations: Pravok — Pravokhettinsky settlement, CUL — cultivar, $\mathrm{x}$ — hybrid, "*” — apophyte.

Range types for each species and plant species belonging to apophytes are given according to Sekretareva (2004). Classification of species into coenotic groups is based on the database of the Institute of Mathematical Problems of Biology of the Russian Academy of Sciences (https:// www.impb.ru/eco/show_info.php?id=1557) (the date of request is 30.11.2020). 


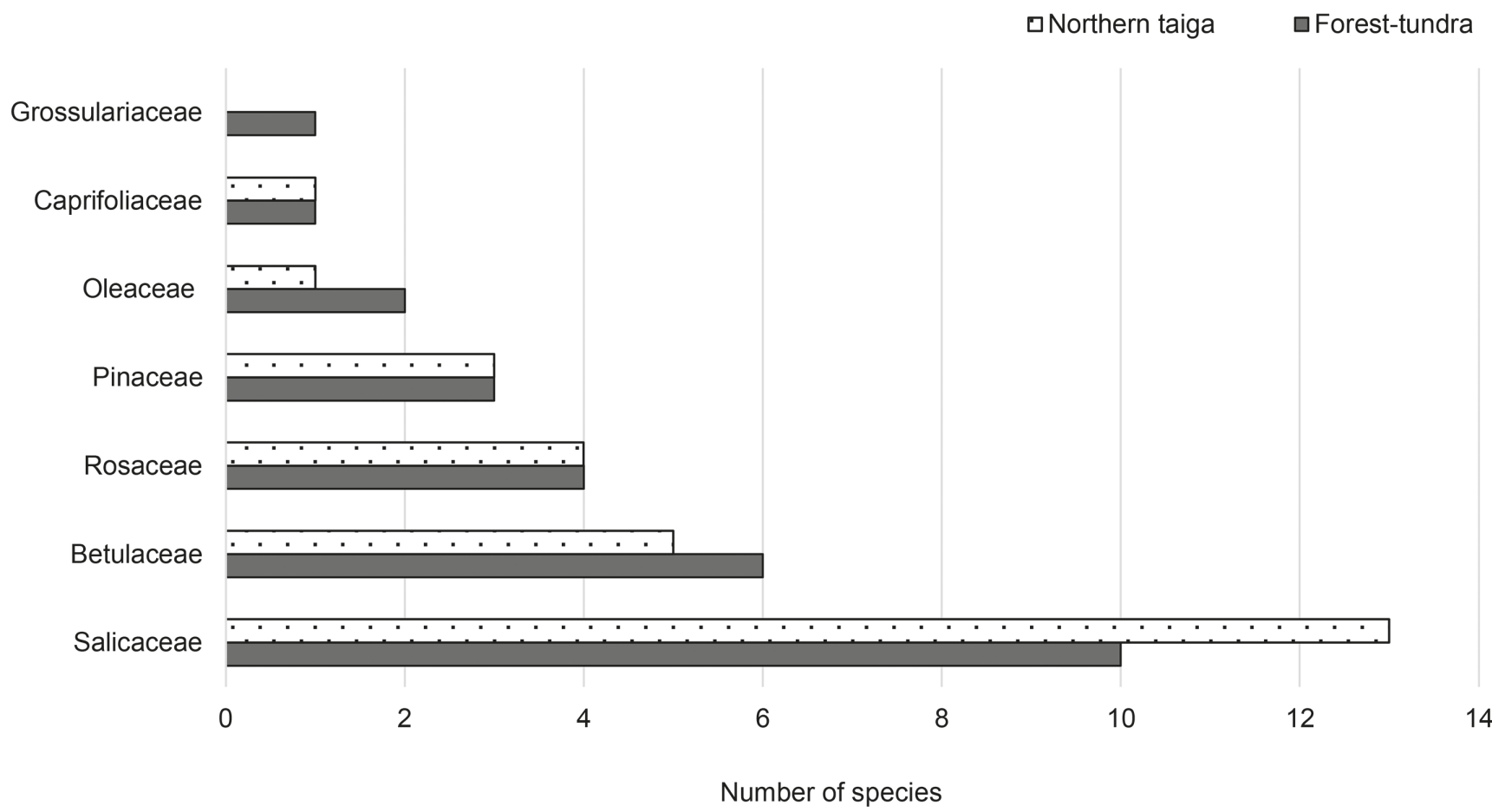

Fig. 1. Distribution by families of woody plants species presented in populated places of northern taiga and forest-tundra.

common in green landscaping. As a rule, these willows were not planted intentionally, but were preserved during construction or colonized new territory by self-seeding.

The difference between functional zones in plant species composition is higher in large and developed towns such as Nadym and Novy Urengoy: the value of Jaccard's index rarely exceeds $30 \%$. In younger and smaller settlements like Pangody and Pravokhettinsky, the similarity between different functional zones within one settlement is significantly higher and up to $40-47 \%$ (Table 3).

Statistical analysis of the sample plot data revealed the differences in vegetation of urban functional zones (Fig. 2). Plant communities of recreational zones, as well as residential neighborhoods or vegetable patches, form isolated clusters. The vegetation of the industrial zone is noticeably more heterogeneous due to the diversity of environmental conditions and various existing negative factors. The similarity of zones in plant species composition is low. Only seven herbaceous plant species (Calamagrostis purpurea (Trin.) Trin., (L.) Schur, Equisetum arvense L., Festuca ovina L., Hieracium umbellatum L., Juncus trifidus L., Taraxacum ceratophorum) are widespread in all types of urban functional zones. Plant species composition in residential neighborhoods is the most diverse, mainly due to weeds and grassland species sown on lawns. Achillea millefolium L., Elymus repens (L.) Gould, Chamaenerion angustifolium, Linaria vulgaris Mill., Melilotus albus Medik., Poa pratensis L.,
Table 3. Similarity of vascular plant species composition between urban functional zones

\begin{tabular}{l|c|c}
\hline Town or settlement & Compared zones & Jaccard's Index value, \% \\
\hline \multirow{4}{*}{ Nadym } & A-B & 25 \\
& B-C & 31 \\
& C-D & 13 \\
& A-C & 20 \\
\hline \multirow{2}{*}{ Novy Urengoi } & A-D & 16 \\
& B-D & 23 \\
\hline \multirow{2}{*}{ Pangody } & A-B & 35 \\
& B-C & 30 \\
\hline Pravokhettinsky & A-C & 23 \\
\hline
\end{tabular}

Note: See Table 2 for functional zone symbols.

Stellaria media (L.) Vill., S.graminea L., Taraxacum ceratophorum (Ledeb.) DC., Tripleurospermum inodorum (L.) Sch. Bip. Vicia cracca L. are the most frequent in this functional zone. The maximum proportion of adventive species $(28 \%)$ is found here.

Some native species typical for taiga forest, such as herbs (Antennaria dioica (L.) Gaertn., Trientalis euro- 


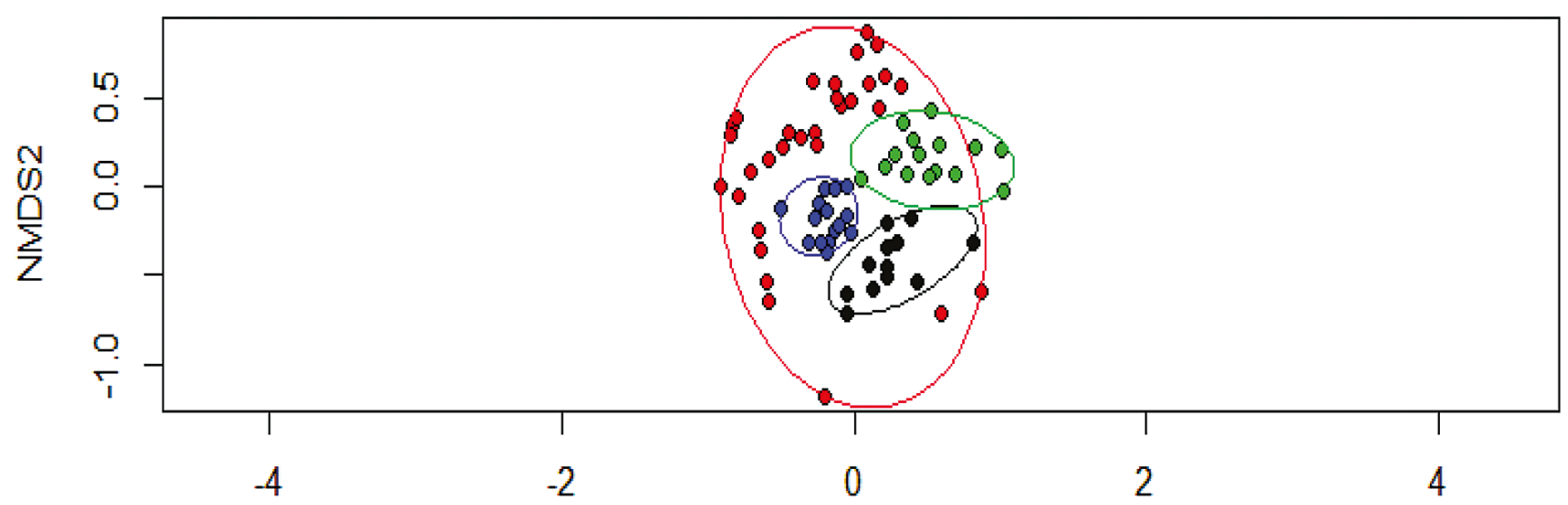

\section{NMDS1}

Fig. 2. Non-metric multidimensional scaling (NMDS) ordination of study plots. Red points - plots in industrial zones; green ones - plots in residential neighborhoods; black — plots in recreation zones; blue — plots in vegetable patches.

paea L., Solidago lapponica With. etc.) and dwarf-shrubs (Arctostaphylos uva-ursi (L.) Spreng., Vaccinium vitisidaea, etc.), are widespread in the recreational zone, because fragments of open larch and birch forests are included in the town parks. The proportion of adventive species in this zone is small $-15 \%$.

The presence of weeds with a clear ruderal strategy is a distinctive feature of industrial zones. Such species as Erigeron acris L., Erysimum cheiranthoides L. and Tussilago farfara L. are common in industrial sites due to the wide spread of eroded sandy soils. Invasive species (Chenopodium album L., Hordeum jubatum L., Polygonum aviculare L., Tripleurospermum inodorum) are noted only in this urban functional zone. Chenopodium album and Polygonum aviculare were also recorded along the roadsides within the towns. However, the share of adventive species in this zone is minimal $-12 \%$.

Field weeds in the vegetable patches are represented by apophyte species. No specific segetal species were found. In total, 20 vascular plant species were recorded in agricultural fields. The small number of recorded weeds is perhaps caused by the small area occupied by vegetable patches. Vascular plants of Asteraceae, Brassicaceae, Chenopodiaceae, and Polygonaceae are the most common in northern agricultural lands.

We have identified both similarities and differences in the ratio of species with different geographic ranges depending on the subzone position of the populated place (Fig. 3). The absolute predominance of Boreal species (more than 60\%) is a characteristic feature for all functional zones of populated places in the northern taiga. Boreal species also lead in the flora of urban functional zones in the forest-tundra but their share is smaller (44-60\%). A large proportion of Boreal species is identified in the residential neighborhoods of cities in both of the above-mentioned subzones due to the use of lawn grass mixtures produced in more southern areas. Graminoids such as Alopecurus pratensis L., Phleum pretense L., Poa pratensis and legumes such as Medicago sativa L., Melilotus albus, Trifolium pratense L. are common on the lawns of residential neighborhoods.

Arctic and Hypoarctic species have significantly smaller proportions. At the same time, the share of Arctic species is the smallest in the most urbanized areas (residential neighborhoods and vegetable patches) in the northern taiga. But cultivars and plurizonal species are maximal in the same zones. The share of Hypoarctic and Arctic species (22 and $34 \%$, respectively) is the maximum only in the recreational zone of the foresttundra town.

Boreal species significantly predominate among woody plants in green spaces of both northern taiga and forest-tundra. Nemoral species (i.e. Syringa $x$ henryi C. K. Schneid. and Syringa josikaea J. Jacq. ex Rchb.f.) are few and alien only. Among woody plants, the number of detected cultivars is approximately the same despite the difference in subzonal position of the study areas (Fig. 4).

The species of urban flora are associated with different types of natural vegetation such as forests, tundra, meadows, etc. (Table 2). Grassland species predominate in all zones of the towns (Fig. 5). The presence of nonurban coenotic groups (forest, tundra, water-swamp) in the flora of northern cities is due to the partial preservation of natural plant communities within the urban landscape. The share of each of these groups is significant and reaches $20-25 \%$. Perhaps this is a distinctive feature of the flora of young northern cities. Cultivars are present only in the urban flora of older and more developed towns; they are not found in Pangody and Pravokhettinsky. 


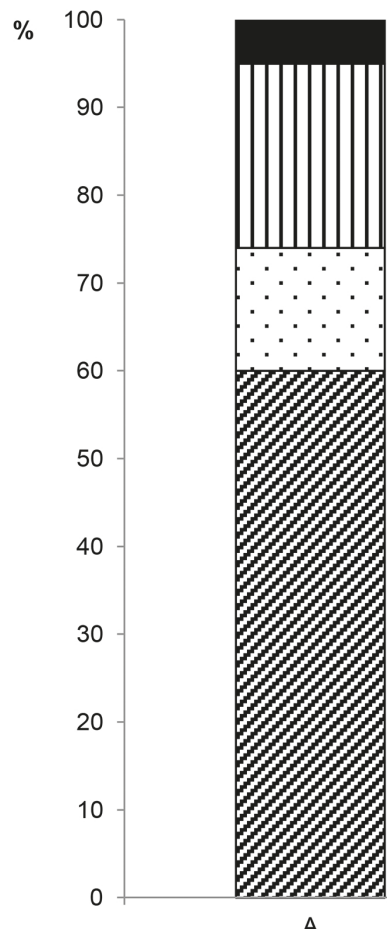

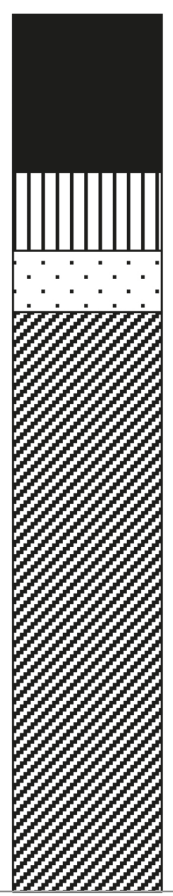

$\mathrm{B}$

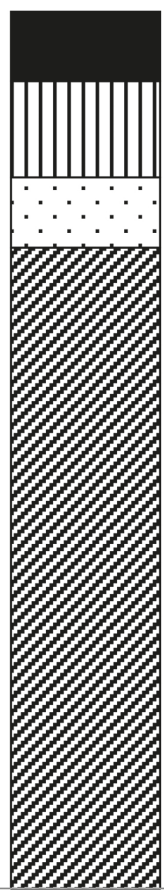

C

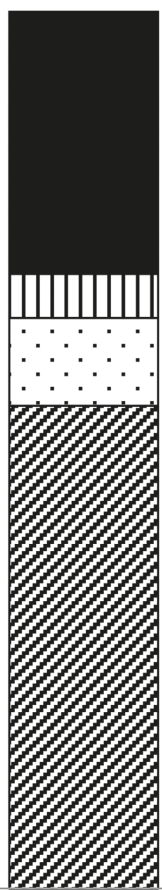

$\mathrm{D}$

A

Functional zone

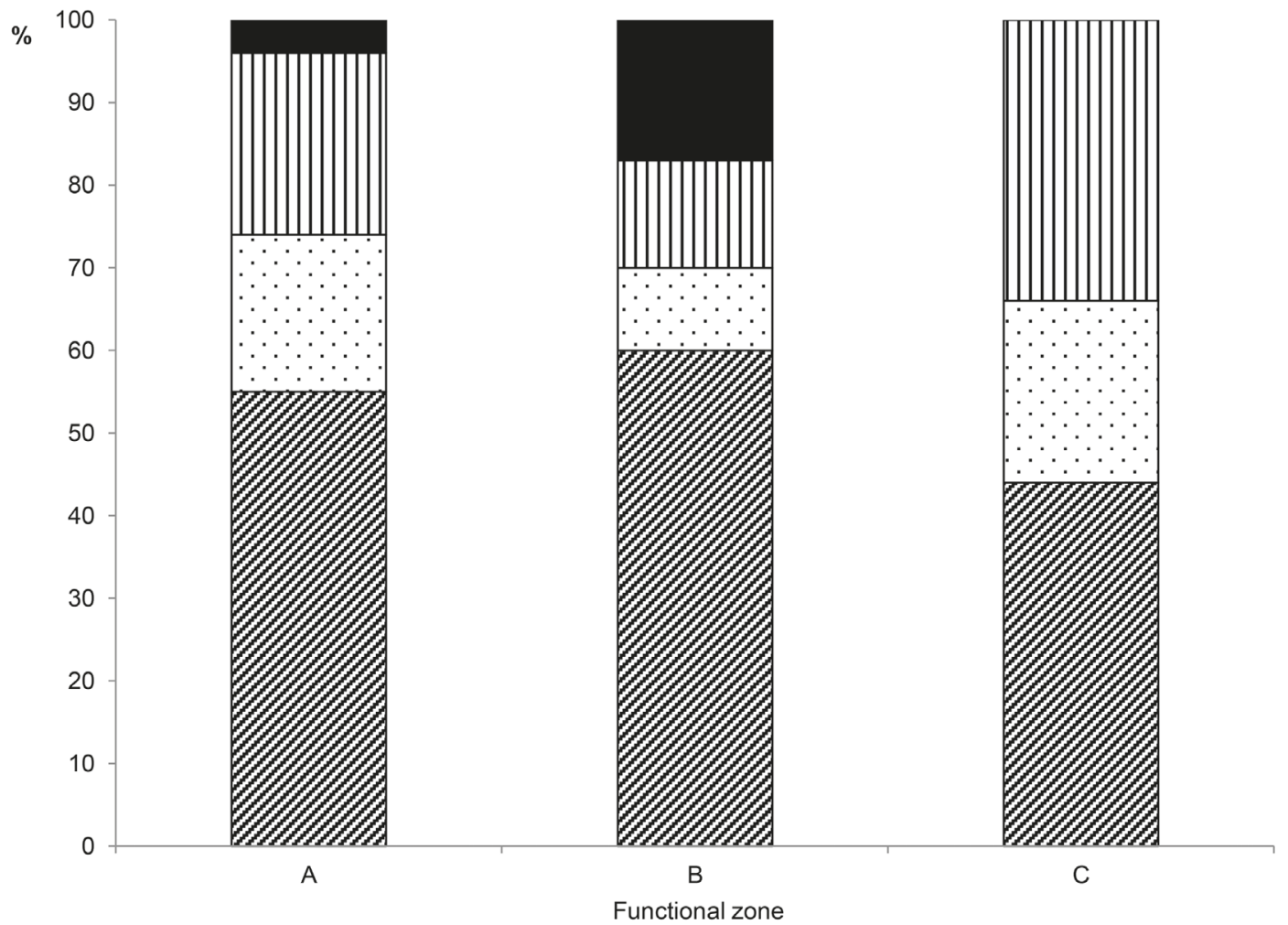

- CUL

DAA

$\square \mathrm{HA}$

GBOR

Fig. 3. The proportion of non-woody plant species with different geographic range in urban functional zones. $a-$ northern taiga, $b-$ foresttundra. A - industrial zones, B - residential neighborhoods, C- recreation zones, D - vegetable patches. BOR - boreal species, HA - hypoarctic species, AA - arctic and arcto-alpine species, CUL — alien cultivars. 


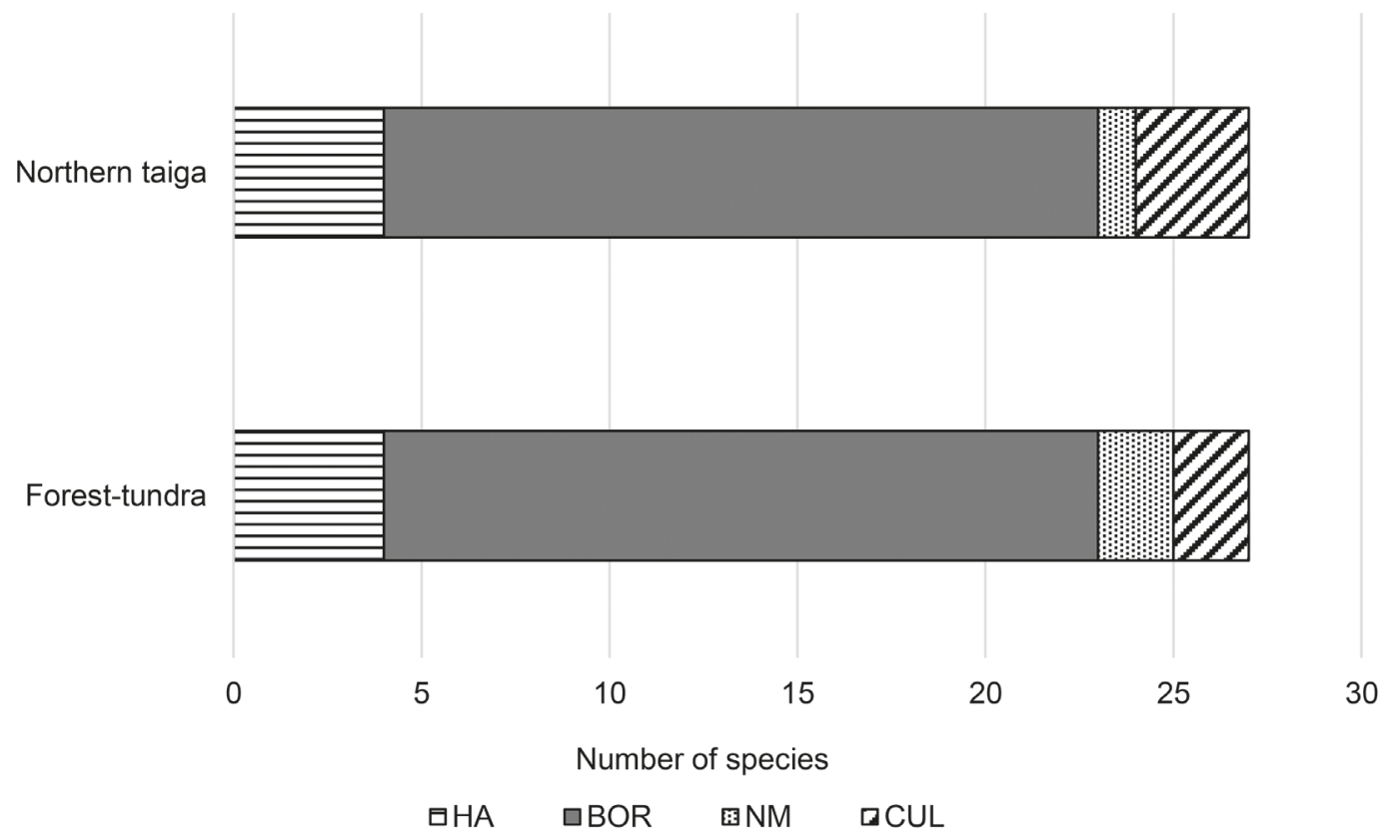

Fig. 4. Woody plant species with different geographical range in populated places of northern taiga and foresttundra.

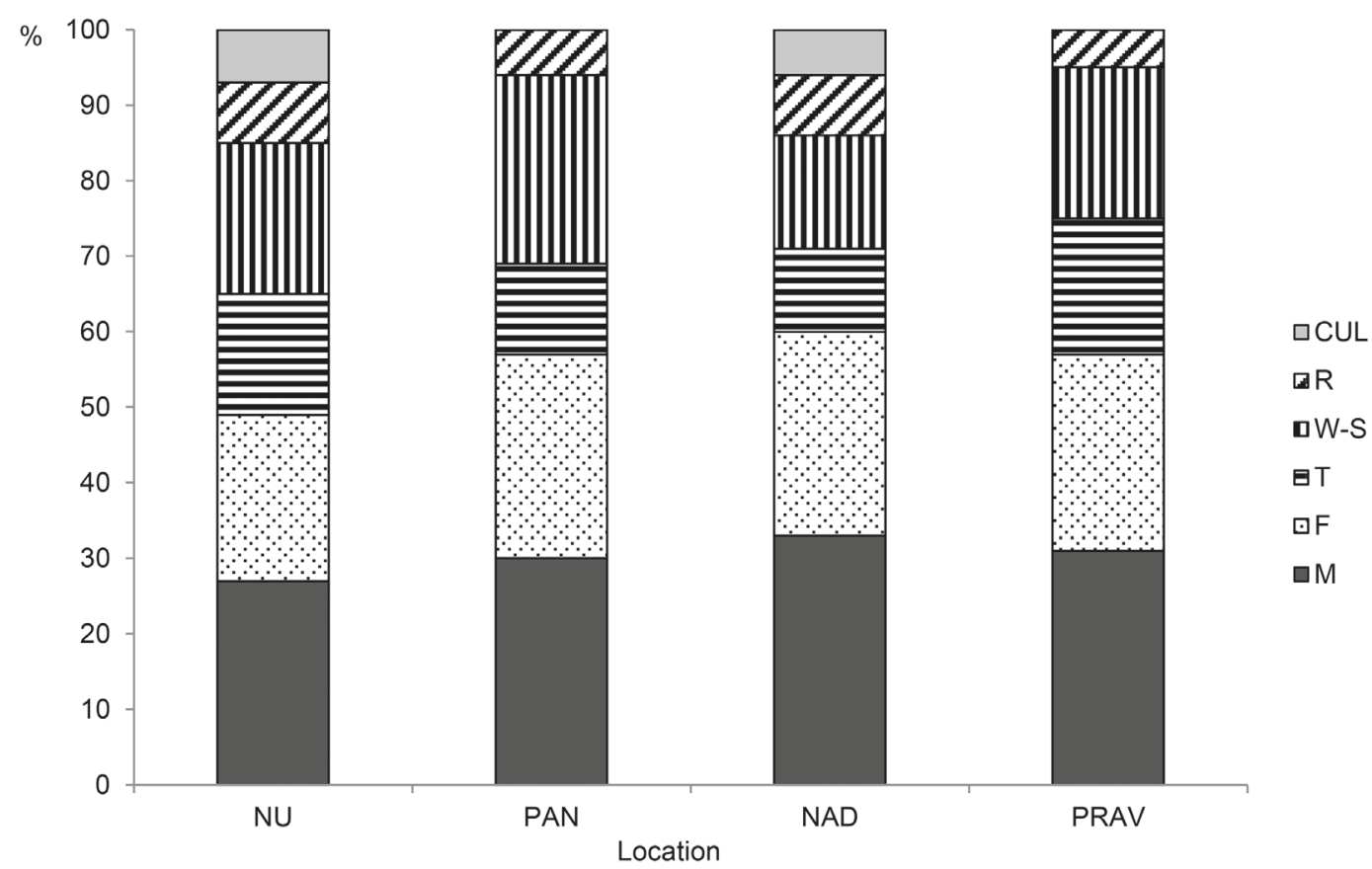

Fig. 5. Groups of species in flora of studied populated places. NU - Novy Urengoy, PAN - Pangody, NAD Nadym, PRAV - Pravokhettinsky. M - Meadow (Grassland) species, F - Forest species, T - Tundra species, W-S - Mire species, R - Ruderal species, CUL - Cultivars.

Glassland species often dominate in the flora of temperate cities (Sudnik-Wojcikowska, 1987; Brandes, 1990). However, we found a different ratio of species by coenotic groups within functional zones in northern populated places (Fig. 6). In our study, grassland species are only leading in the residential neighborhoods and in vegetable patches but forest species are most common in parks belonging to the recreational zone. The noticeable proportion $(25 \%)$ of plants from natural disturbed sites is a specific feature of vegetable patches. The landscaping in industrial zones is the worst. So, there are many wet puddles that are occupied by water-swamp plant species. Cultivars are especially typical for residential neighborhoods and recreational sites of populated places. 


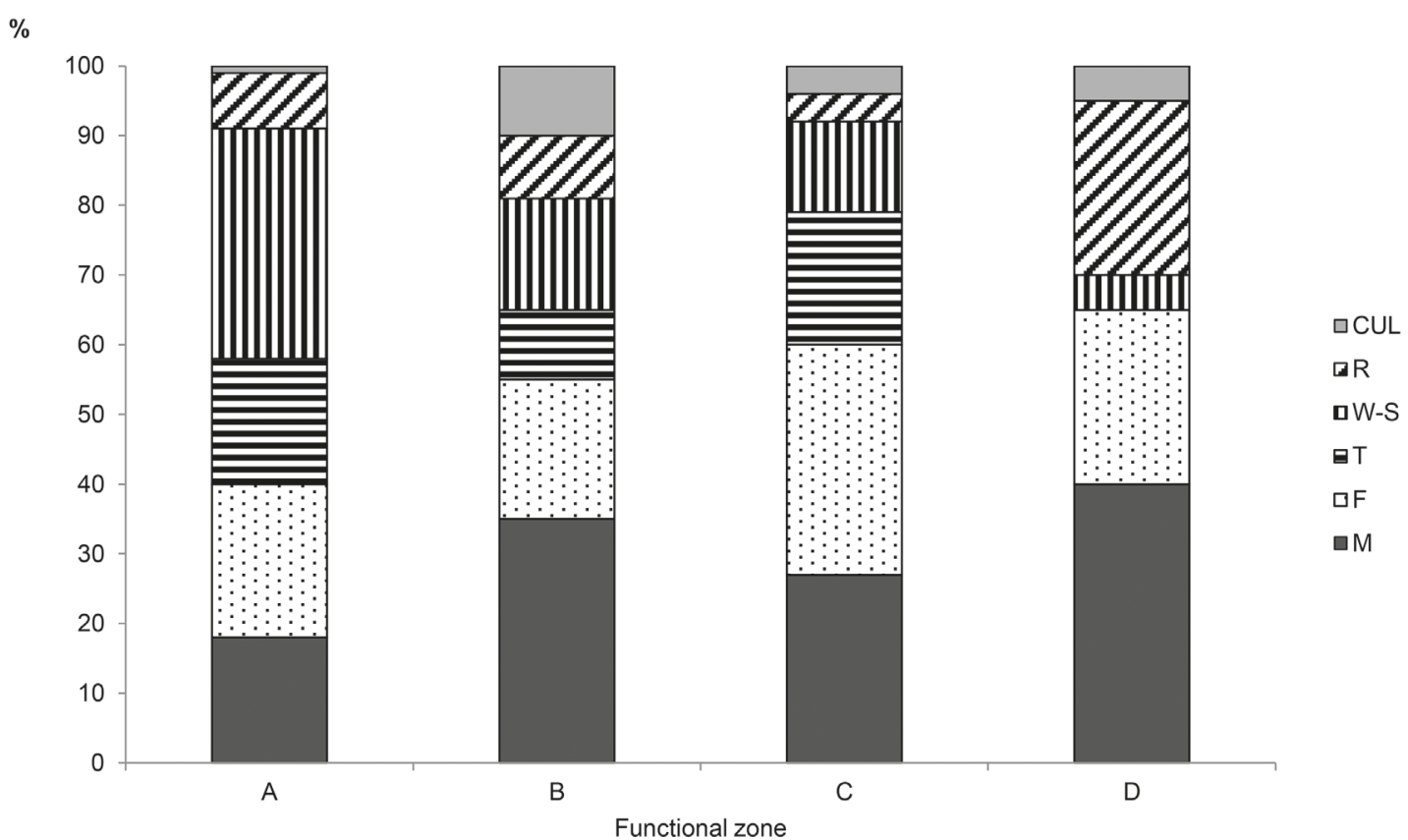

Fig. 6. Groups of species in different functional zones. A - industrial zones, B - residential neighborhoods, C- recreation zones, D - vegetable patches. M - Meadow (Grassland) species, F — Forest species, T — Tundra species, W-S Mire species, R - Ruderal species, CUL — Cultivars.

\section{Dynamics of urban flora}

Based on published data (Vilchek and Kuznetsov, 1996; Sumina, 2013), we revealed the dynamics of urban flora in Novy Urengoy and examined its trends. The first list of urban flora dates back to 1995 and contains information about 95 species of vascular plants found in Novy Urengoy (Vilchek and Kuznetsov, 1996). After 23 years, some cultivars (i.e. Helianthus annuus L.) or species from southern areas (i.e. Poa nemoralis L.) are not recorded in the town. Perhaps their invasion was one-shot, and these species were not able to acclimatize later in the northern environment. Some native species (i.e. Ledum palustre, Linnaea borealis L., Pyrola minor L., Rubus arcticus Card, R. chamaemorus L., Vaccinium myrtillus, etc.) were also recorded by us in 2018 even in the town parks.

Plant species diversity in different functional zones is still growing. For example, in 2018 we counted 90 plant species in Novy Urengoy in residential neighborhoods, which is $21 \%$ more than data recorded by Vilchek and Kuznetsov (1996) in 1995. Similarly, $18 \%$ more species were observed in 2018 in the industrial zone of this town. The species composition in urban functional zones changed significantly over time. The comparison of vascular plant lists for residential neighborhoods and industrial sites of Novy Urengoy obtained by Vilchek and Kuznetsov (1996) with our data via the Jacquard index showed values of $34 \%$ and $33 \%$, respectively.

In 1993-1994, the flora and vegetation on technogenic sites in the vicinity of Novy Urengoy were stud- ied in detail by Sumina (2013). This allowed us to make a pairwise comparison of all functional zones of Novy Urengoy with technogenic sites in its vicinity using data on the species composition of plants. As it turned out, the flora of technogenic sites is closest to the urban industrial zone. The Jacquard index was about $40 \%$, while for other urban zones it was less than $30 \%$.

\section{Discussion}

Urbanization is a global process which is expanding from year to year, so urban ecology is becoming a fastdeveloping field of scientific study. Generalized and detailed information on urban biodiversity and ecological processes is mostly connected with European cities and towns (Niemelä et al., 2011); thereby any data on urban ecology of Siberian localities situated in northern areas are valuable. Our results have shown some specific traits of floristic diversity in all study towns and settlements of the northern taiga and forest-tundra.

Unlike in most southern cities, the urban flora of northern localities is poor and native species form its basis. The low diversity of vascular plants, which is typical even to natural vegetation, can be explained by the low diversity of habitats and large areas with sandy grounds on the West Siberian Plain (Vilchek and Kuznetsov, 1996).

As a rule, the richness of urban flora depends on alien invasive and cultivated species (Pyšek, 1998), and 
they are represented mostly by introduced woody plants (Salinitro et al., 2018). In northern areas, where urban greening uses the resources of the local flora, species of trees and shrubs are not numerous and belong only to two families (Salicaceae and Rosaceae).

Herbs predominate in the list of species of all studied populated places, and the Poaceae family is represented by the largest number of species. Such a high role of graminoids could be connected with the wide area of disturbed territories and bare grounds (Sumina, 2013) in young and developing northern towns.

In northern towns, alien species are represented generally by a small number of individuals. Severe climate and poor soils limit the alien plants' abilities to establish in the Far North. However, the situation may change if the climate becomes warmer in the future. The potential dangers of invasive species have been highlighted in many publications (Clemants and Moore, 2003; Altay and Özyiit, 2010). The advent of new species in northern cities has been discussed in recently published data (Byalt, Pismarkina and Egorov, 2017). The appearance of alien decorative forbs (Dianthus barbatus L., Papaver somniferum L., etc.), shrubs (Rosa glabrifolia C. A. Mey. ex Rupr.), spicy herbs (Carum carvi L., Coriandrum sativum L., Origanum vulgare L., etc.), as well as poisonous and dangerous plants (i.e. Conium maculatum L.) has been noted (Byalt, Pismarkina and Egorov, 2017). However, most of these plants are annual in northern Russia and need special care to survive. Some herbs (i.e. Lolium perenne L. and Medicago falcata L.) penetrate into urban flora via lawn grass seed mixtures.

The high level of vegetation unlikeness between temperate cities due to the heterogeneity of habitats and varied opportunities for plant immigration into urban territories has been noted in many publications (Sukopp and Werner, 1983; Pyšek, 1989, 1993; Kühn, Brandl and Klotz, 2004; Schmidt, Poppendieck and Jensen, 2014). In our results, the flora of studied towns and settlements were quite similar, especially in woody plant composition, despite the difference in latitudinal position (in northern taiga and forest-tundra). Perhaps this similarity can be explained by the similar climatic conditions as well as the wide ecological amplitude of apophyte species which are able to colonize different urban habitats.

Segmentation of urban territory into functional zones clarifies the processes of the formation of urban flora in northern localities. Different functional zones differ notably from each other in terms of plant species diversity. Plant species composition in industrial zones, where the lowest number of adventive species was noted, is the closest to native flora. Residential neighborhoods, unlike others urban zones, have a high vascular species diversity mainly due to cultivars; this trait was also noted for temperate cities (Threlfall et al., 2016). This richness is partly connected with spontaneous par- ticipation of residents in greening. Recreational zones of northern towns with parks, which contain fragments of former open forests with numerous native species, are notably differing from similar sites in temperate cities. In the studied vegetable patches in Nadym there are not specific segetal plants, and all field weeds are apophyte species from the local flora.

Our results demonstrate some difference between four urban functional zones by vegetation, but this is only the beginning of their differentiation. No trend of unified urban vegetation was revealed. Other researchers (Pearse et al., 2018) also failed to collect data confirming their hypothesis about urban flora homogenizing. Species diversity of urban flora in northern areas depends on the size and age of the populated place; in developed and large towns the flora is more diverse than in younger and smaller settlements.

The long history of European towns gives an opportunity to analyze the role of alien species in urban vegetation for more than a century (Kowarik, 2008). In young northern towns we can observe the start of such a process.

The increasing diversity of vascular species in northern towns is partly connected with woody plant introduction. A large number of woody taxa and cultivars of the Rosaceae family and some other families unusual to the area, for example Oleaceae (Syringa ssp.), are planted spontaneously due to residents' desire to improve the aesthetic appearance of their localities. Gradual replacement of native species from the Salicaceae family by alien families' species which are more decorative or brightly flowering is observed in northern greening. The use of fertilizers and mulching in vegetable patches has a positive influence on boreal species' survival. The tendency of boreal species' fraction increasing is also revealed in recreational zones thanks to lawn grass sowing.

At the same time, it is necessary to realize that urbanization promotes the reduction of indigenous biodiversity (Thompson and Jones, 1999), but in the northern regions where native species has the adaptive advantage, this effect could be minimized. A feature of the formation of flora in most northern cities is the initial preservation of native species, including herbaceous and especially woody plants. Nonetheless, some Arctic and Arcto-Alpine species are particularly sensitive to conditions of urban 'heat islands'. The amplitude of land surface temperature anomalies in some cities of Northwest Siberia corresponds to climates in areas located $300-600 \mathrm{~km}$ to the south (Esau, 2016). The influence of the 'heat island' effect on the flora of northern cities has not been sufficiently studied. In this regard, urban parks formed on the basis of natural vegetation are of particular importance for the successful conservation of Arctic and Arcto-Alpine species. 


\section{Conclusion}

The analysis of urban flora in four populated places of Northwest Siberia contributes to the understanding of current trends in the development of northern cities' green spaces. Plant species diversity is higher in larger towns, expectedly. Perennial herbaceous plants form the basis of urban vegetation. At the same time, the share of alien woody plants used in gardening of northern towns is insignificant. The formation of the species composition is affected by the partial preservation of natural vegetation in urban areas. As a result, northern green spaces are dominated by native species of grasses and woody plants, most of which are apophytes. The proportion of apophyte species often exceeds $50 \%$ in most functional zones, but the share of alien cultivars is the smallest.

Unlike cities in the temperate zone, there was no tendency to unify urban flora in northern towns or settlements. Significant differences in urban flora were found not only between towns, but also between functional zones within the same town. Species composition in the functional zones of the studied cities turned out to be specific. In terms of species composition, the functional zones of bigger towns (Novy Urengoy and Nadym) are more contrasting than in younger and less populated places (Pangody, Pravokhettinsky). Residential neighborhoods stand out for their wide plant diversity, especially in big towns.

Detectable changes in the diversity of urban species composition over the past 20-25 years prove that the process of the formation of urban flora, even in large cities, cannot be considered complete or stable. The main dynamic processes are associated less with an increase, but rather with a change in the composition of plant species. In the future more explicit differentiation in plant species composition between different functional zones of any town could be expected. Nowadays many changes in urban flora are mostly caused by the spontaneous activity of residents. For the successful green development of northern cities, it is necessary to control and manage the processes of their vegetation development; this process should be well-planned and deliberate.

\section{Acknowledgments}

The authors thank the staff of the Research Center of the Yamal-Nenets Autonomous District.

\section{References}

Altay, V., Özyii, B. I., and Yarci, C. 2010. Urban flora and ecological characteristics of the Kartal District (Istanbul): A contribution to urban ecology in Turkey. Scientific Research and Essays 5:183-200.

Atlas of the Yamal-Nenets Autonomous District. 2004. Omsk. (In Russian)

Brandes, D. 1995. The flora of old town centers in Europe; pp. 49-58 in Sukopp, H. et al. (Eds.) Urban ecology as the basis of urban planning. Amsterdam.
Byalt, V. V., Pismarkina, E. V., and Egorov, A. A. 2017. New findings of alien vascular plant species in the YamalNenets Autonomous district. Botanicheskii Zhurnal 102:1663-1682. (In Russian)

Clemants, S. E. and Moore, G. 2003. Patterns of species diversity in eight Northeastern United States cities. Urban Habitats 1:4-16.

Esau, I. and Miles, V. 2016. Warmer urban climates for development of green spaces in northern Siberian cities. Geography, Environment, Sustainability 9:48-61. https:// doi.org/10.24057/2071-9388-2016-9-4-48-62

Flora of vascular plants in the Central European Russia. The database of the Institute of mathematical problems of Biology of the Russian Academy of Sciences. https:// www.impb.ru/eco/

Gogoleva, P. A., Cherosov, M. M., and Pavlova, Z. S. 1990. Syntaxonomy of ruderal vegetation of Yakutsk city. Nauchnye doklady vysshej shkoly. Biologicheskie nauki 8:116123. (In Russian)

Hao, P. Y., Dong, L., Zhang, C. H., Zhou, X. L., Feng, B., Huang, L., Ma, Y. N., Dong, Z. H., Zheng, Y. M., and Zhang, N. 2013. The composition and characteristics of urban plants and tree communities in green spaces attached to urban roads, Beijing, China. Acta Hortic 999:69-79. https://doi. org/10.17660/ActaHortic.2013.999.9

International Plant Names Index (IPNI). https://www.ipni.org

Ishbirdin, A. R. 2004. Ecological and geographical regularities of synanthropic flora of Russia. II. The analysis of regional synanthropic flora. Botanicheskii Zhurnal 89:1456-1469. (In Russian)

Knapp, S., Kühn, I., Stolle, J., and Klotz, S. 2010. Changes in the functional composition of a Central European urban flora over three centuries. Perspectives in Plant Ecology, Evolution and Systematics 12:235-244. https://doi. org/10.1016/j.ppees.2009.11.001

Kowarik, I. 2008. On the role of alien species in urban flora and vegetation; pp. 321-338 in Marzluff, J. M. et al. (Eds.) Urban Ecology. Springer, Boston, MA. https://doi. org/10.1007/978-0-387-73412-5_20

Kühn, I., Brandl, R., and Klotz, S. 2004. The flora of German cities is naturally species rich. Evolutionary Ecology Research 6:749-764.

Mavimbela, L. Z., Sieben, E. J. J., and Procheş, S. 2018. Invasive alien plant species, fragmentation and scale effects on urban forest community composition in Durban, South Africa. New Zealand Journal of Forestry Science 48:19. https://doi.org/10.1186/s40490-018-0124-8

Niemelä, J., Breuste, J. H., Elmqvist, T., Guntenspergen, G., James, P., and Mclntyre, N.E. 2011. Urban Ecology: Patterns, Processes, and Applications. Oxford University Press. https://doi.org/10.1093/acprof:o so/9780199563562.001.0001

Pearse, W. D., Cavender-Bares, J., Hobbie, S. E., Avolio, M. L., Bettez, N., Chowdhury, R.R., Darling, L.E., Groffman, P. M., Grove, J. M., Hall, S., Heffernan, J. B., Learned, J., Neill, C., Nelson, K. C., Pataki, D. E., Ruddell, B. L., Steele, M. K., and Trammell, T. L. E. 2018. Homogenization of plant diversity, composition, and structure in North American urban yards. Ecosphere 9:e02105. https://doi.org/10.1002/ecs2.2105

Pyšek, P. 1989. On the richness of Central European urban flora. Preslia 61:329-334.

Pyšek, P. 1993. Factors affecting the diversity of flora and vegetation in central European settlements. Vegetatio 106:89-100. https://doi.org/10.1007/BF00044860

Pyšek, P. 1998. Alien and native species in Central European urban floras: a quantitative comparison. Journal of Biogeography 25:155-163. https://doi.org/10.1046/j.13652699.1998.251177.x 
Riordan, B., Verbyla, D., and McGuire, A. D. 2006. Shrinking ponds in subarctic Alaska based on 1950-2002 remotely sensed images. JGR: Biogeosciences 111:G04002. https:// doi.org/10.1029/2005JG000150

Rozhdestvenskiy, J.F. 2000. About the possibility of green space in the Far North, the Urals and Western Siberia. Materials to knowledge of fauna and flora of the Yamal-Nenets Autonomous District (part II), Salekhard, pp. 70-74. (In Russian)

Salinitro, M., Alessandrini, A., Zappi, A., Melucci, D., and Tassoni, A. 2018. Floristic diversity in different urban ecological niches of a southern European city. Scientific Reports 8:15110. https://doi.org/10.1038/s41598-018-33346-6

Schmidt, K. J., Poppendieck, H. H., and Jensen, K. 2014. Effects of urban structure on plant species richness in a large European city. Urban Ecosyst 17:427-444. https://doi. org/10.1007/s11252-013-0319-y

Sekretareva, N. A. 2004. Vascular plants of the Russian Arctic and adjacent territories. Moscow. (In Russian)

Shushannikova, G. S. 2001. The synanthropic flora of the taiga and tundra areas of the North-East of European Russia. Botanicheskii Zhurnal 86:28-37. (In Russian)

Smith, L. C., Sheng, Y., MacDonald, G. M., and Hinzman, L. D. 2005. Disappearing Arctic lakes. Science 308:1429. https://doi.org/10.1126/science.1108142

Srodnykh, T. B. 2008. Growth and development under the influence of zonal and intrazonal factors of woody species prevailing in urban greening of the northern cities of West Siberia. Agrarian Herald of Urals 8:79-83. (In Russian)

Sudnik-Wojcikowska, B. 1987. Flora miasta Warszawy i jej przemiany w ciągu XIX i XX wieku. Część I. Warszawa, pp. 3-242.

Sukopp, H. and Werner, P. 1983. Urban environment and vegetation; pp. 247-260 in Holzner, W., Werger, M .J. A., and Ikushima, I. (Eds.), Man's impact on vegetation, The Hague.

Sumina, O. I. 2013. Formation of vegetation on technogenic habitats of the Russian Far North. Saint-Petersburg. (In Russian)

Thompson, K. and Jones, A. 1999. Human population density and prediction of local plant extinction in Britain. Conservation Biology 13:185-189. https://doi.org/10.1046/ j.1523-1739.1999.97353.x

Threlfall, C. G., Ossola, A., Hahs, A. K., Williams, N. S. G., Wilson, L., and Livesley, S. J. 2016. Variation in vegetation structure and composition across urban green space types. Frontiers in Ecology and Evolution 4:1-12. https:// doi.org/10.3389/fevo.2016.00066

Trotsenko, G. V. 1990. Synanthropization of flora of Labytnangi, in structure, productivity and dynamics of vegetation. Sverdlovsk, pp. 101-110. (In Russian)

Uchida, T., Xue, J.H., Hayasaka, D., Arase, T., Haller, W.T., and Gettys, L. A. 2014. The relation between road crack vegetation and plant biodiversity in urban landscape. International Journal of GEOMATE 6:885-891. https://doi. org/10.21660/2014.12.3177

Vila-Ruiz, C.P., Meléndez-Ackerman, E. J., Santiago-Bartolomei, R., Garcia-Montiel, D., Lastra, L., Figuerola, C. E., and Fumero-Caban, J. 2014. Plant species richness and abundance in residential yards across a tropical watershed: implications for urban sustainability. Ecology and Society 19:22. https://doi.org/10.5751/ES-06164-190322

Viltchek, G. E. and Kuznetsov, D. V. 1996. Flora of anthropogenic habitats in the vicinity of Novy Urengoy city; pp. 101-121 in Viltchek, G. E., Sumina, O. I., and Tishkov, A. A. (Eds.) Flora of anthropogenic habitats of the North, Moscow. (In Russian) 